\title{
Des versants aux masses d'eau : érosion, colmatage et envasement
}

\author{
Rosalie VANDROMME ${ }^{1}$, Olivier EVRARD ${ }^{2}$, Thomas GRANGEON ${ }^{1,3}$ \\ Olivier CERDAN ${ }^{1}$, Sébastien SALVADOR-BLANES ${ }^{3}$
}

\author{
${ }^{1}$ BRGM-e-mail: rvandromme@brgm.fr \\ ${ }^{2}$ CEA-CNRS-UVSQ \\ ${ }^{3}$ Université François Rabelais, E.A 6293 GéHCo
}

Mots-clés : versants, masses d'eau, érosion, colmatage

\section{From hillslopes to rivers: erosion, clogging and silting}

Key words: hillslopes, rivers, erosion, clogging

Les paysages français ont subi de profonds bouleversements ces dernières décennies. Qu'il s'agisse du reboisement de versants montagneux, de la rectification des cours d'eau ou du remembrement du parcellaire agricole, ces modifications ont des conséquences directes sur la nature et l'intensité des transferts sédimentaires. En amont, notamment sur les versants cultivés, l'érosion a été identifiée comme étant l'une des principales menaces pesant sur les sols par la Commission Européenne (Montanarella, 2015). Lorsqu'elle prend la forme de ravines, elle peut directement gêner l'exploitation des parcelles cultivées par les agriculteurs. En mobilisant les particules les plus fines ainsi que les nutriments, elle menace également, de manière plus insidieuse, les rendements agricoles à plus long terme (Bakker et al., 2005). Lorsque les sédiments transportés par le ruissellement aboutissent dans les cours d'eau en aval des versants cultivés, les problèmes qu'ils génèrent sont fortement amplifiés. Certains sont immédiats, comme les coulées de boue qui causeront des dégâts sur le réseau routier ou dans les habitations riveraines. D'autres effets seront plus durables et ils conduiront notamment au colmatage et à l'envasement des étangs, des retenues et de certains tronçons de cours d'eau. Le colmatage peut ainsi être défini comme étant le dépôt de sédiments très fins, d'origine minérale ou organique, sur le lit des rivières, impactant directement les habitats aquatiques.

Les processus qui régissent ces phénomènes sont cependant complexes, ce qui s'illustre par exemple au travers des très grandes difficultés actuelles à atteindre les objectifs de rétablissement de la continuité écologique et sédimentaire, et plus largement de la qualité des milieux aquatiques, fixés par la Directive Cadre européenne sur l'Eau (DCE). C'est pour tenter de faire un tour d'horizon des points de vue de différents acteurs jouant un rôle au sein de cette problématique qu'a été organisée à Orléans une journée de séminaires et d'échanges intitulée « Des versants aux masses d'eau : érosion, colmatage et envasement ». Cette journée a réuni plus de 100 participants qui ont interagi avec les orateurs ayant abordé les différents aspects qui doivent être pris en compte pour une étude intégrée de l'érosion des versants et du colmatage des cours d'eau.

Dans un premier temps, les aspects techniques et les questions scientifiques ont été abordés. Ainsi, à l'échelle du bassin versant, l'analyse détaillée des relations entre le débit et la charge sédimentaire des cours d'eau permet de mieux appréhender la dynamique des sources et des stocks de sédiments dans les bassins versants (Crave, ce numéro). Le développement de techniques de traçage sédimentaire permet aussi de reconstruire la contribution de ces différentes sources (comme celle de la surface des sols cultivés par opposition à celle des berges) aux flux sédimentaires (Foucher et al., ce numéro). Plus à l'aval, l'impact des barrages et des autres retenues devient prépondérant. La gestion de ces ouvrages a alors un impact considérable sur les flux sédimentaires, tant pour le charriage des matériaux grossiers que pour le transfert des fines (Camenen et al., ce numéro ; Malavoi et al., ce numéro).

La qualification du colmatage en tant que tel à l'échelle d'un tronçon de cours d'eau donné et, a fortiori, à l'échelle d'un grand bassin hydrographique, pose tout une série de problèmes pratiques, mais elle permet néanmoins d'identifier les zones nécessitant une attention particulière de la part des gestionnaires (Dupeau et Favreau, ce numéro). Des expérimentations concrètes montrent en effet qu'il est possible de limiter les apports de particules provenant de l'amont des bassins versants, à partir d'aménagements d'hydraulique douce (Ouvry et Ledun, ce numéro). Les gestionnaires ont ainsi pu émettre, dans un second temps, leur point de vue sur ce qu'est le colmatage et sur les manières de le limiter.

Les différentes communications et échanges ont permis de générer une prise de conscience tant au sein de la communauté des chercheurs que de celle des gestionnaires sur la nécessité d'étudier de concert les processus survenant sur les versants et ceux qui interviennent dans les rivières. Cette connexion entre l'érosion sur les versants cultivés et le transfert et le dépôt de sédiments dans les cours d'eau situés en aval n'a pas été suffisamment étudiée par la communauté scientifique, car elle nécessite de mettre autour de la table des experts issus de différentes disciplines qui ne communiquent pas systématiquement. Les pédologues et les agronomes travaillent bien souvent sur les seuls versants cultivés, tandis que les rivières sont le site d'étude de prédilection des hydrauliciens, les sédimentologues se concentrant souvent sur l'accumulation de matières dans les zones réceptacles que sont par exemple les lacs et les étangs. On retrouve cette même déconnexion du côté des gestionnaires. Les sols cultivés sont en effet plutôt gérés par le Ministère de l'Agriculture, tandis que les cours d'eau le sont par les agences de bassin qui dépendent du Ministère de 
l'Environnement (qui s'appelle aujourd'hui le Ministère de la Transition Ecologique et Solidaire).

Il est également ressorti de cette journée un manque de connaissances dû à la faible disponibilité de réseaux pérennes de suivi et de mesure des flux hydrique et sédimentaire dans les paysages et les cours d'eaux. À l'avenir, il conviendra donc de développer de nouvelles méthodes permettant progressivement de s'orienter vers une quantification du colmatage dans les cours d'eau. De tels développements permettront d'améliorer nos connaissances sur la genèse de ce phénomène et d'orienter au mieux les mesures de gestion qui pourraient être prises pour le limiter au maximum, ceci afin de recouvrer le bon état écologique des cours d'eau. Ces mesures nécessiteront néanmoins de combiner des actions et des aménagements, tant à l'amont des bassins versants, qui sont les principales zones productrices de sédiments, que dans les cours d'eau à l'aval, qui en sont les principaux réceptacles. Une telle gestion intégrée à l'échelle du bassin versant constituerait alors le pendant " sédimentaire " de la gestion à l'échelle des districts hydrographiques voulue par la DCE.

\section{RÉFÉRENCES}

Bakker M.M., Govers G., Kosmas C., Vanacker V., Oost K.V., Rounsevell (2005) - Soil erosion as a driver of land-use change. Agriculture, Ecosystems \& Environment, 105, 467-481.

Montanarella L. (2015) - Agricultural policy: Govern our soils. Nature, 528, 32-33.

http://www.brgm.fr/evenement/versants-masses-eau-erosioncolmatage-envasement 